www.jmscr.igmpublication.org Impact Factor 5.244

Index Copernicus Value: 83.27 ISSN (e)-2347-176x ISSN (p) 2455-0450 crossref DOI:_http://dx.doi.org/10.18535/jmscr/v4i7.09

Journal Of Medical Science And Clinical Research

\title{
Direct detection of Left Ventricular Diastolic Dysfunction by Phase image analysis in Gated Thallium SPECT Scan
}

\author{
Authors \\ Dr V.Sivasubramaniyan ${ }^{1}$, Dr K. Venkataramaniah ${ }^{2}$ \\ ${ }^{1}$ Doctoral Research Scholar, ${ }^{2}$ Senior Professor, \\ Dept of Physics, Sri Sathya Sai Institute of Higher Learning, Vidhyagiri, \\ Prasanthinilayam, Anantapur Dist. A.P 515134
}

\begin{abstract}
Aim: The evaluation and differentiation of Left Ventricular Diastolic Dysfunction has a vital role in the management of heart failure patients. Currently the diastolic dysfunction is inferred from the $E$ and $A$ wave changes occurring in m-mode echocardiographic changes at the mitral orifice mostly. In the gated myocardial perfusion imaging from the time volume curve the Peak Flow Rate and Time to Peak Flow rate are derived and assessed for the presence and extent of diastolic dysfunction. All these parameters represent the changes that are occurring due to the underlying diastolic dysfunction in the left ventricular muscle mass. For the first time this study aims to highlight the diagnosis of Left Ventricular Diastolic Dysfunction by the visual Phase image analysis in Gated Thallium SPECT Scan. The presence of inhomogenous, irregular and insufficient tracer accumulation in the Diastolic phase images with the normal and homogenous tracer distribution in the Systolic Phase images is proposed to indicate the diastolic dysfunction. It also thrives to quantitate the changes observed by calculating the $S / D$ ratio by dividing the Systolic Counts by the diastolic counts obtained from the raw phase images. It is correlated with echocardiographic stages of the Diastolic Dysfunction.

Materials and Methods: This study consists of 75 Males in the age group of 28 to 63 years with mean age of $47.9+/-7.8$ years and 25 Females in the age range of 32 to 55 years with mean age of $49+/-6.8$ years. After exercising the patients on a bicycle ergometer using Bruce Protocol $2 \mathrm{mCi}$ of Thallum-201 was injected intravenously Gated SPECT MPI were acquired using Siemens Dual Head E-Cam Gamma Camera. The analysis was done using GATED SPECT PERFUSION ANALYSIS Protocol. The S/D ratio was calculated using the region ratio count protocol in the ICON software.

Results: The discordance between the Diastolic and systolic images were found in 98/100 (98\%)Image1,2. The Echocardiographic evidence of Diastolic dysfunction as classified into Grade II and grade III using E / A ratio analysis was found in 87/100 (87\%). The diabetes was seen in 16/100 (16\%) and Hypertension was encountered in 63/100 (63\%). The S/D ratio was $1.27+/-0.32$ in Grade IILVDD and $1.68+/-0.03$ Grade III LVDD.

Conclusion: In conclusion for the first time the utility of direct visual individual phase image analysis in the detection of Left Ventricular Diastolic Dysfunction is highlighted. The Scintimetric Characterization of the same with the help of S/D ratio shown it to be different between stage II and stage III LVDD.

Keywords: Gated Thallium SPECT, Left Ventricular Diastolic Dysfunction, echocardiography, Scintimetric evaluation, S/D Ratio.
\end{abstract}




\section{Introduction}

The diastolic function of the left ventricle has a vital role in the preservation and efficacy of the left ventricular systolic function. Hence, the evaluation of left Ventricular Diastolic function is of much concern in the diagnosis and management of the left heart failure. Routinely it is evaluated by the M-mode echocardiography at the mitral valve orifice from the tracing $\mathrm{E} / \mathrm{A}$ ratio is calculated. The E / A ratio of $<0.8$ is considered to represent Stage I, $>1.4$ in Stage II and $>1.8$ in Stage III left ventricular Diastolic dysfunction ${ }^{(1,2)}$. Further refining of these parameters by introducing the Color Doppler echocardiography and tissue characterization methods also have been described ${ }^{(3)}$.The advent of ECG- Gated SPECT studies has enabled us to have greater insights into its evaluation. The calculation of Ejection Fraction and visual analysis of Regional Wall Motion Abnormalities by the Gated SPECT Myocardial Perfusion Studies are well known and most utilized. However, the portal of analyzing and extracting useful information from the Phase image analysis of diastolic and systolic phases of the cardiac cycle remains unexplored. After Morgan and Mannting (4) described the utility of Gated SPECT Myocardial Perfusion Imaging in 1993 it was Raymond Taillefer ${ }^{(5)}$ et al described the utility of Diastolic Image analysis in the early detection of C.A.D in women as against Summed image analysis in 1999. Siemens has developed a GATED SPECT PERUFSION PROCESSING protocol based on the work of Depuey et al ${ }^{(6)}$ which includes separate image analysis in Diastole and Systole, Ejection fraction calculation and Regional Wall Thickness assessment. Utilising Phase image analysis in the Gated SPECT Perfusion processing the incidence of In-homogenous, Irregular and Insufficient Tracer distribution in the diastolic images in the presence of Normal homogenous, regular and sufficient uniform tracer distribution in the Systolic summed images is inferred to indicate the presence of diastolic dysfunction. Its association with the echocardiographic diastolic dysfunction markers particularly E/A ratio and Diabetes Mellitus \& Hypertension is also highlighted in our presentation in SNMICON ${ }^{(7)}$. The good correlation between the Peak Flow rate and S/D ratio calculated using the Time Volume curves obtained from the ECG- gated SPECT and the E / A ratio had been documented ${ }^{(8)}$. The normal values of the diastolic dysfunction parameters like Peak Flow Rate and Time To Peak Flow have been reported in the assessment of diastolic Function Using 16Frame 99mTc-Sestamibi Gated Myocardial Perfusion SPECT with total agreement between the QGSPECT and the Echocardiographic assessment ${ }^{(9)}$. In their experience with 16-gated Myocardial Perfusion SPECT assessment of Diastolic Heart Function RD Lele et.al have reported that diastolic dysfunction was diagnosed in 92 / 121 (76\%) and only 53/121 (43\%) by echocardiographic E / A ratio analysis ${ }^{(10)}$.

\section{Aims}

This prospective study aims to highlight the (i) utility of summed image analysis of the Diastolic and Systolic phases of the Cardiac cycle in the visual diagnosis of the Left Ventricular diastolic dysfunction and (ii) to quantitatively differentiate between the different echocardiographic stages of the LVDD as reported in the echocardiography report.

\section{Materials and Method}

The Left ventricular Diastolic Dysfunction evaluation study consists of 35 males in the age range of 31 to 67 years with mean age of $51.9+/-7.4$ years and 12 females in the age range of 31 to 55 years with mean age of 45.9 $+/$ - 6.6 years. After exercise the patients on a Treadmill using Bruce Protocol $2 \mathrm{mCi}$ of Thallum-201 was injected intravenously Gated SPECT MPI were acquired using Siemens Dual Head E-Cam Gamma Camera. The images were analysed using the ICON Software GATED 


\section{JMSCR Vol||4||Issue||07||Page 11226-11233||July}

SPECT PERFUSION ANALYSIS Protocol. The presence of in-homogenous, irregular and insufficient tracer distribution in the diastolic phase images as comparedto the normal Systolic phase images denote the diastolic dysfunction changes in the left ventricualr muscle tissue as shown in Fig.1

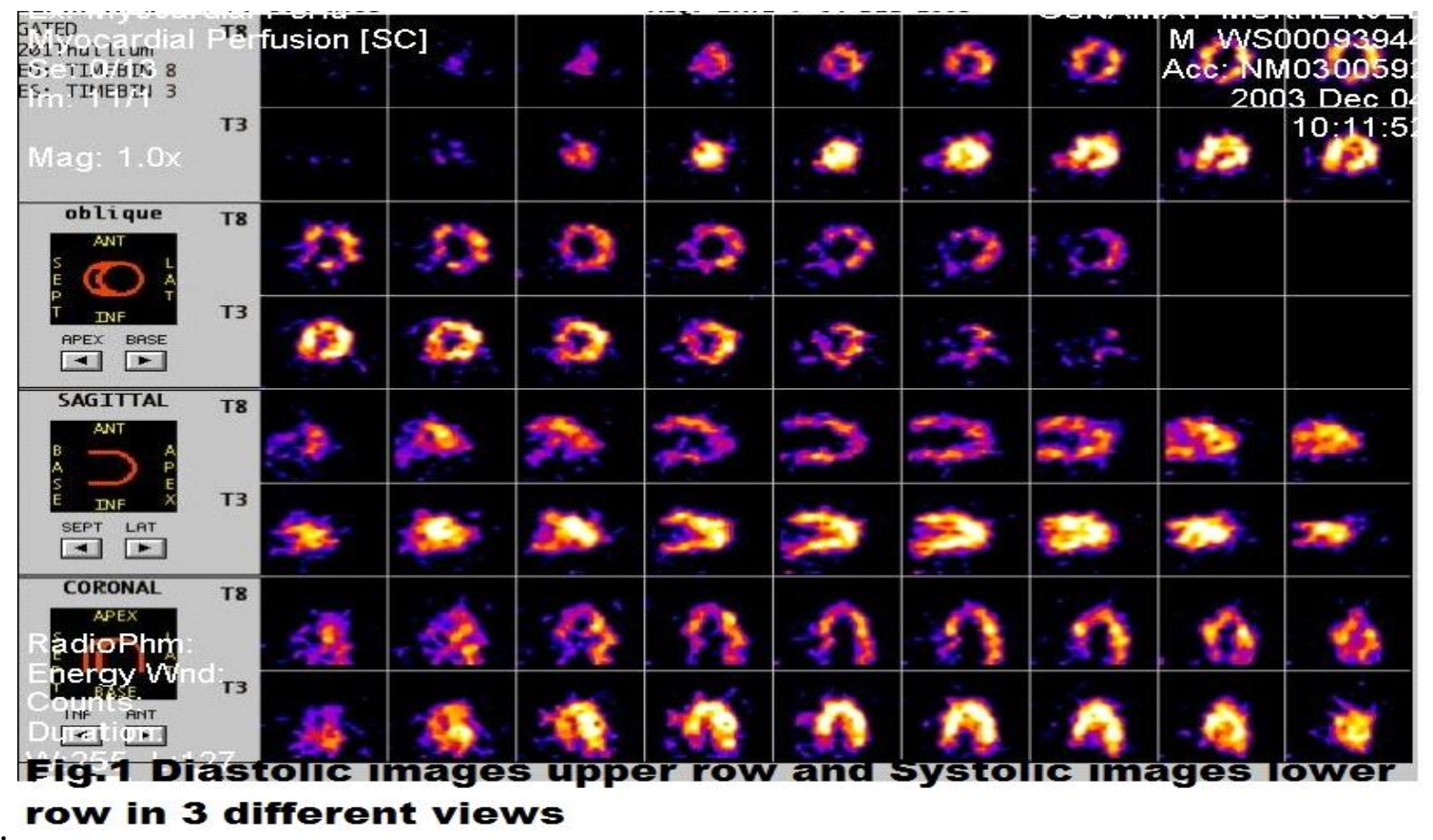

The upper row diastolic phase images in different views show consistently decreased, inhomogeneous, insufficient and irregular tracer distribution indicative of perfusion deficiency in them. The lower row systolic phase images show normal and uniform tracer distribution. This finding of discordance between the diastolic and systolic phase images isattributed to direct changes occurring in the left ventricular muscle mass due to diastolic dysfunction. These images are subjected to left ventricular ejection fraction and Bull's eye map analysis of the end diastolic perfusion, end systolic perfusion and wall thickening estimation as shown in Fig.2.

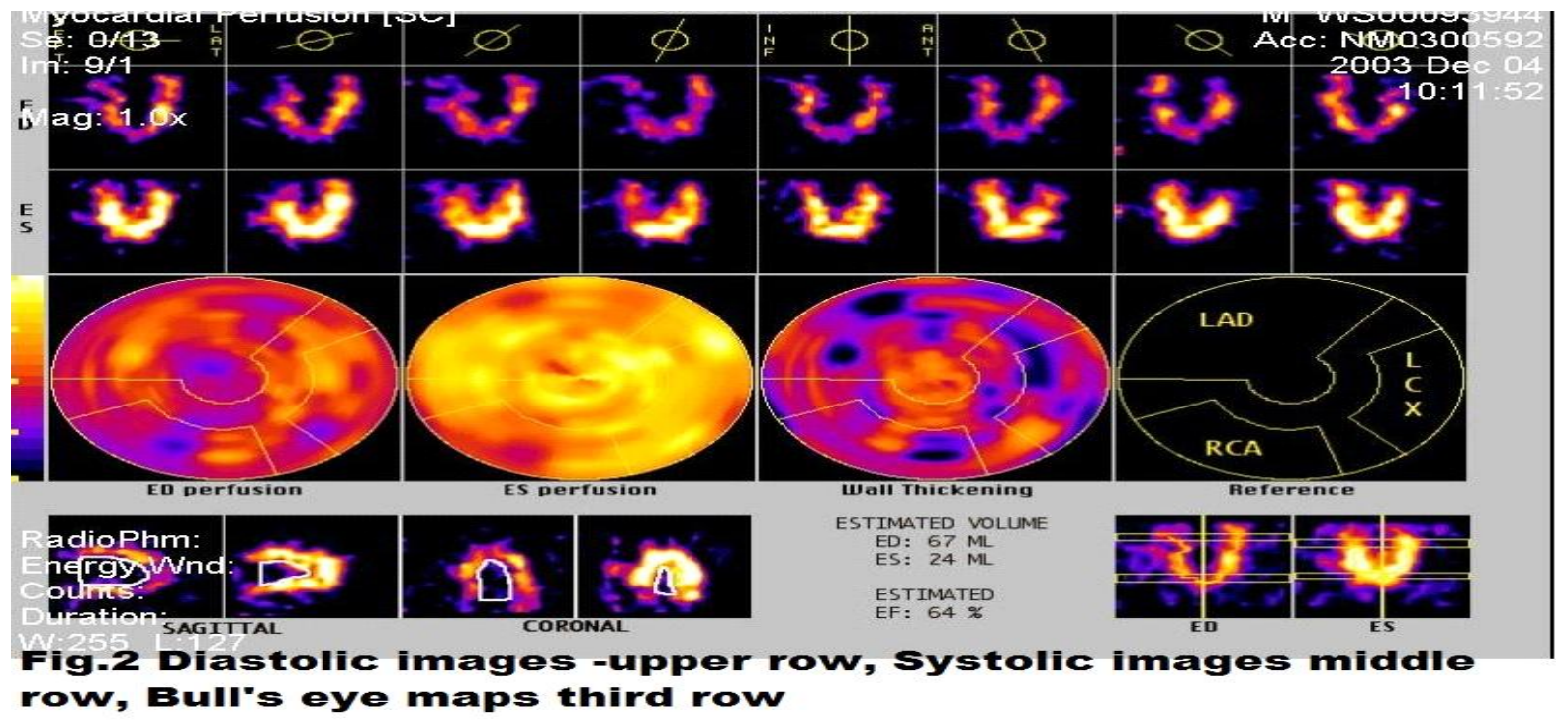


The upper and lower row images represent the diastolic and systolic phase images respectively. The diastolic phase ED perfusion bull's eye map shows moderate to severe affliction as per the colour scale and the systolic phase ES perfusion bull's eye map shows normal state as per the colour scale. The calculated left ventricular ejection from the derived end diastolic ED and end systolic ES volumes is in the normal range.

The findings are noted down. The echocardiographic grading of the left ventricular diastolic dysfunction is also tabulated along with the above findings from the case records. The image data sets of the diastolic and systolic images in the S.A, H.L.A \& V.L.A views were saved separately. Then the Ventricular wall outline is outlined by drawing their outer and inner margins in the representative Diastolic and Systolic phase images and the counts in the region of interest were calculated using the region ratio count protocol in the ICON software Fig. 3.

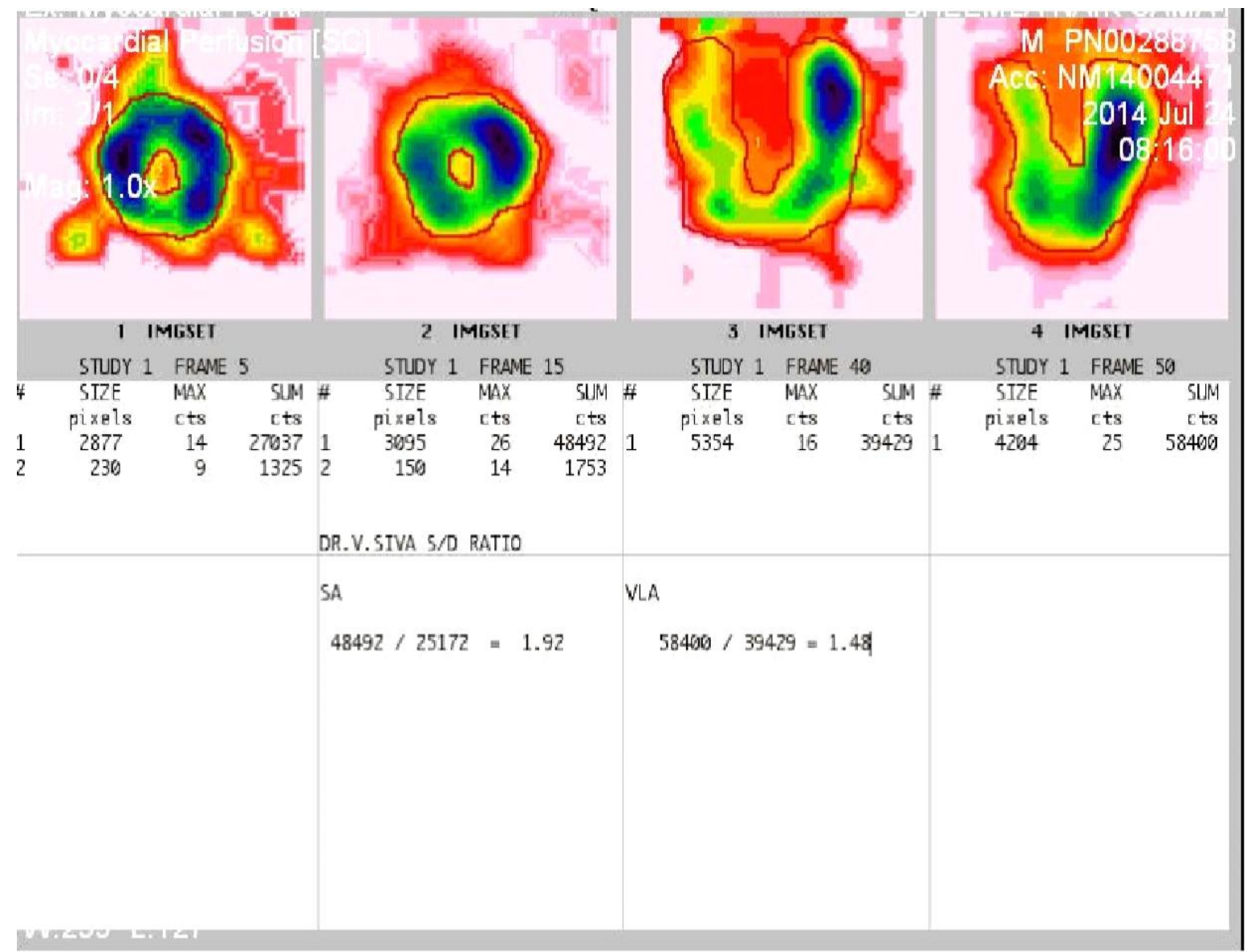

Fig.3 Scintimetric method of calculating Distolic and Systolic for deriving S/D ratio

The systolic and diastolic counts encountered in the male and female patients of the study were tabulated along with the derived S/D ratio in the
Fig.4 and Fig 5. The S/D ratios belonging to stage ii and stage iii diastolic dysfunction also were analysed separately. 
Fig.4 Male S/D ratio data sheet

\begin{tabular}{|c|c|c|c|c|c|c|c|}
\hline NAME & $\mathrm{SC}$ & DC & S/D RATIO & NAME & $\mathrm{SC}$ & DC & S/D RATIO \\
\hline \multirow[t]{4}{*}{ ANILKUMAR } & 13419 & 6769 & 1.98 & & 19668 & 13460 & 1.48 \\
\hline & 13521 & 9211 & 1.46 & & 38404 & 23476 & 1.6 \\
\hline & 16515 & 12546 & 1.31 & & 47748 & 3396 & 1.4 \\
\hline & 25603 & 20473 & 1.25 & KKDAS & 14439 & 7395 & 1.95 \\
\hline \multirow[t]{4}{*}{ BANNERJEE D } & 34937 & 18552 & 1.88 & & 15693 & 11794 & 1.33 \\
\hline & 45910 & 34152 & 1.34 & & 27614 & 18876 & 1.46 \\
\hline & 17198 & 6617 & 2.5 & & 39516 & 24819 & 1.57 \\
\hline & 26876 & 18718 & 1.43 & K REDDY & 156815 & 116886 & 1.31 \\
\hline \multirow[t]{4}{*}{ BNAIK SAMAY } & 48492 & 25172 & 1.92 & & 171959 & 134118 & 1.28 \\
\hline & 58400 & 39429 & 1.48 & M S JENA & 40351 & 24731 & 1.63 \\
\hline & 24193 & 10058 & 2.4 & & 31292 & 24296 & 1.28 \\
\hline & 28534 & 16194 & 1.76 & M K PAUL & 29543 & 20595 & 1.47 \\
\hline \multirow[t]{4}{*}{ CHNADRA O R } & 61932 & 56177 & 1.1 & & 43270 & 38179 & 1.13 \\
\hline & 62994 & 52708 & 1.19 & & 14412 & 8082 & 1.7 \\
\hline & 27944 & 17141 & 1.68 & & 18307 & 11724 & 1.56 \\
\hline & 22057 & 17887 & 1.2 & M MUSTAFA & 38279 & 29289 & 1.3 \\
\hline \multirow[t]{4}{*}{ DASTAGIRI } & 33712 & 23280 & 1.44 & & 32827 & 29797 & 1.1 \\
\hline & 30622 & 25351 & 1.2 & & 12554 & 6138 & 2 \\
\hline & 12011 & 6711 & 1.7 & & 9605 & 6060 & 1.58 \\
\hline & 9024 & 5812 & 1.5 & N BABU & 64466 & 39641 & 1.62 \\
\hline \multirow[t]{4}{*}{ DS } & 42773 & 33265 & 1.28 & & 85114 & 63104 & 1.34 \\
\hline & 32785 & 30254 & 1.08 & NR & 25894 & 12819 & 2.01 \\
\hline & 29478 & 19755 & 1.49 & & 36390 & 26415 & 1.37 \\
\hline & 23188 & 17578 & 1.32 & & 24239 & 11760 & 2.06 \\
\hline \multirow[t]{4}{*}{ B DAS } & 18086 & 12770 & 1.41 & & 30287 & 15318 & 1.97 \\
\hline & 16279 & 13864 & 1.18 & OPSAU & 32845 & 20908 & 1.4 \\
\hline & 35562 & 25653 & 1.38 & & 34947 & 23183 & 1.5 \\
\hline & 31553 & 25120 & 1.25 & & 13153 & 7053 & 1.8 \\
\hline \multirow[t]{4}{*}{ GPAL } & 13713 & 6246 & 2.1 & & 26213 & 19258 & 1.36 \\
\hline & 11669 & 6800 & 1.7 & & & & \\
\hline & 17533 & 13901 & 1.26 & & & Mean & 1.535967742 \\
\hline & 16940 & 9149 & 1.8 & & & variance & 0.205 \\
\hline \multirow[t]{4}{*}{ G BARIK } & 12052 & 6788 & 1.7 & & & Std.devi & 0.4528 \\
\hline & 19668 & 13460 & 1.48 & & & & \\
\hline & 38404 & 23476 & 1.6 & & & & \\
\hline & 47748 & 3396 & 1.4 & & & & \\
\hline
\end{tabular}

Fig 5 Female $S$ / D ratio data sheet

\begin{tabular}{|c|c|c|c|c|}
\hline N A M E & SYST COUNTS & DIAST COUNTS & S / D RATI0 & \\
\hline FAKRU BI & 16492 & 7892 & 2.08 & \\
\hline & 16273 & 13864 & 1.3 & \\
\hline & 32753 & 19595 & 1.67 & \\
\hline & 26920 & 20528 & 1.3 & \\
\hline J DEVI & 29991 & 20138 & 1.48 & \\
\hline & 28423 & 21395 & 1.32 & \\
\hline & 29893 & 19334 & 1.5 & \\
\hline
\end{tabular}




\begin{tabular}{|c|c|c|c|c|}
\hline & 27827 & 21338 & 1.3 & \\
\hline JBWGUM & 24890 & 19944 & 1.23 & \\
\hline & 27359 & 20180 & 1.35 & \\
\hline & 75524 & 51525 & 1.46 & \\
\hline & 79245 & 72761 & 1 & \\
\hline J BEE BI & 24483 & 11766 & 2 & \\
\hline & 37403 & 25799 & 1.4 & \\
\hline & 50888 & 29066 & 1.75 & \\
\hline & 67414 & 56117 & 1.2 & \\
\hline Kondamma & 15524 & 6950 & 2.2 & \\
\hline & 20853 & 12953 & 1.6 & \\
\hline & 54093 & 30278 & 1.78 & \\
\hline & 69373 & 36734 & 1.88 & \\
\hline Shantamma & 23730 & 21457 & 1.1 & \\
\hline & 40885 & 30719 & 1.3 & \\
\hline & 32524 & 22433 & 1.44 & \\
\hline & 49253 & 34719 & 1.41 & \\
\hline & & Mean & 1.502083 & \\
\hline & & variance & 0.1389 & \\
\hline & & standard deviation & 0.3726 & \\
\hline & & & & \\
\hline
\end{tabular}

\section{Results}

The discordance between the Diastolic and systolic images were found in 98/100 (98\%). The Echocardiographic evidence of Diastolic dysfunction as classified into Grade ii and grade iii using E/ A ratio analysis was found in 87/100

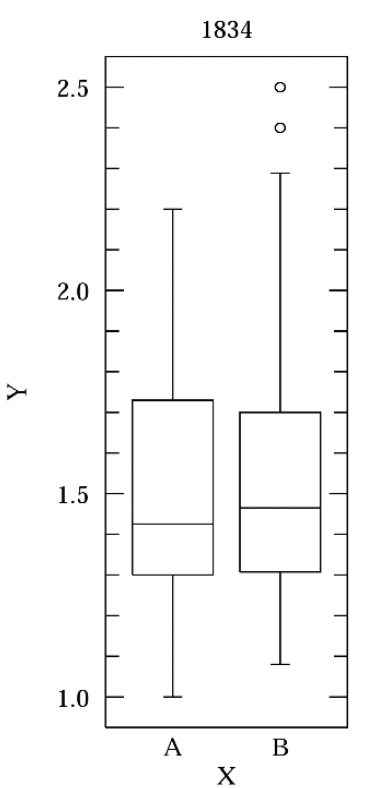

Fig.6 No difference between A-Male \& B- Female Scintimetric S/D Values

The S/D ratio was $1.47+/-0.32$ in Grade II LVDD and $1.81+/-0.03$ Grade III LVDD. The statistical analysis of the data with Paired Student t-test
(87\%). The diabetes was seen in 16/100 (16\%) and Hypertension was encountered in $63 / 100$ (63\%). The S/D ratio values between the male and female group did not show any significant statistical difference -Fig.6.

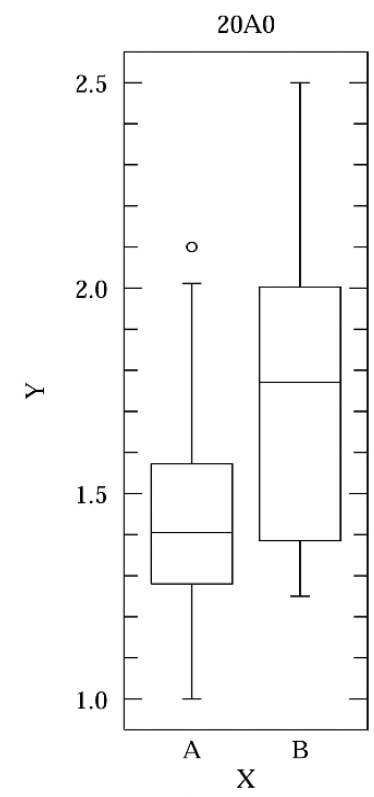

Fig.7 Significant difference between A- Stage ii and $B-$ Stage iii Scintimetric S/D values

confirmed the statically significant difference between the S/D ratio values between the Stage ii and Stage iii grades - Fig.7. 


\section{Discussion}

This is the first article highlighting the utility of the visual phase image analysis of the diastolic phase images and the systolic phase images in the identification and interpretation of the left ventricular diastolic dysfunction of the heart.By this method 98/100 (98\%) of the patients with diastolic dysfunction were identified. Whereas only 87/100(87\%) of patients were identified by $\mathrm{E}$ / A echocardiographic examination. The visual phase image analysis of detecting the diastolic dysfunction is based on the direct changes occurring in the left ventricular cardiac myocytes hence were able to diagnose the diastolic dysfunction in as high as $98 \%$ in our series.In their experience with 16-gated Myocardial Perfusion SPECT assessment of Diastolic Heart Function RD Lele et.al using time volume curve analysis were able to diagnose 92/121 (76\%) only (10). Whereas the direct visual image interpretative assessment of diastolic and systolic phase images resulted in the detection of diastolic dysfunction as high as $98 \%$. However the study should be extended to all remaining the gated SPECT studies to cover larger population.The separate individualized comparison of the ED and ES Perfusions in both stress and rest gated myocardial perfusion imaging findings should also be contemplated. Since this study group did not include normal population a study in normal control group must also be contemplated.

\section{Conclusion}

Conclusively it can be said that this method of assessing the radiotracer distribution in the diastolic phase of the cardiac cycle by evaluating the changes occurring in the myocardial muscle tissue directly and correlating it with the presence of Diastolic dysfunction is unique and highly useful in the early detection of the same. The addition of scintimetric quantitation by S/D count ratio to this method will aid in the differentiation of various stages of the left ventricular diastolic dysfunction as well.

\section{References}

1. Recommendations for the Evaluation of Left Ventricular Diastolic Function by Echocardiography -Sherif F. Nagueh, MD, Chair†, Christopher P. Appleton, MD†, Thierry C. Gillebert, MD*, Paolo N. Marino, MD*, Jae K. Oh, MD†, Otto A. Smiseth, MD, PhD*, Alan D. Waggoner, MHS $\dagger$, Frank A. Flachskampf, MD, CoChair*, Patricia A. Pellikka, MD $\dagger$, and Arturo Evangelisa, MD* Houston, Texas; Phoenix, Arizona; Ghent, Belgium; Novara, Italy; Rochester, Minnesota; Oslo, Norway; St. Louis, Missouri; European Journal of Echocardiography (2009) 10, 165-193

2. A Guideline Protocol for the Echocardiographic assessment of Diastolic Dysfunction D rThomas Mathew (leadauthor) Dr Rick Steeds, Chair Dr Richard Jones Dr Prathap Kanagala Dr Guy Lloyd Dr Daniel Knight Dr KevinO' Gallagher Dr David Oxborough Dr Bushra Rana Dr Liam Ring Julie Sandoval Gill Whart on Dr Richard Wheeler Published November2013

3. Evaluation of Left Ventricular Diastolic Function by Echocardiography Juan Lacalzada et al.* Hospital Universitario de Canarias, La Laguna, Santa Cruz de Tenerife Chapter 6, Establishing Better Standards of Care in Doppler Echocardiography, Computed Tomography and Nuclear Cardiology Edited by Dr. Richard M. Fleming ,2011.

4. Gated SPECT with Tc99m SESTAMIBI for assessment of Myocardial Perfusion abnormalities, Morgan M.G., Mannting.F et.al JNM 1993:21;13

5. Comparison between End Diastolic and the Summed images of Gated Tc $99 \mathrm{~m}$ MIBI SPECT in the detection of C.A.D in women -Raymond Taillefer, E.GordonDupey et.al JNC, March/April1999 
6. Left Ventricular Ejection fraction assessed from Gated Tc99m sestamibi SPECT - Depuey.E.G, Nicholas K et al JNM, $1993: 34$ :

7. Role of Gated SPECT Myocardial Perfusion Imaging in the Evaluation of Diastolic Dysfunction of the Heart V. Sivasubramaniyan, P. K. Dash, V. R. Iyer, R. D. Prasad, N. Purantharan. Oral Paper Presentation Session 1 (Parallel) (CardioVascular System) Abstracts of SNMICON 2003.

8. Left ventricular systolic/diastolic function evaluated by quantitative ECG-gated SPECT: comparison with echocardiography and plasma BNP analysis Ichiro NAKAE, ${ }^{*}$ Shinro MATSUO, $*$ Terue $\mathrm{KOH}, *$ Kenichi MITSUNAMI** and Minoru HORIE* Annals of Nuclear Medicine Vol. 19, No. 6, 447-454, 2005.

9. Assessment of Diastolic Function Using 16Frame 99mTc-Sestamibi Gated Myocardial Perfusion SPECT: Normal Values CigdemAkincioglu, MD1; Daniel S. Berman, MD1,2; Hidetaka Nishina, MD1; Paul B. Kavanagh, PhD1; Piotr J. Slomka, PhD1,2; Aiden Abidov, MD1; Sean Hayes, MD1; John D. Friedman, MD1,2; and Guido Germano, PhD1,2 J Nucl Med 2005; 46:1102-1108

10. Assessment of Diastolic Heart Function Experience with 16-gated Myocardial Perfusion SPECT RD Lele, KarunaLuthra, Yogini Sawant JAPI • VOL. 56 • 763-767, OCTOBER 2008. 